EPiC Series in Engineering
Volume 3, 2018, Pages 852-857
HIC 2018. 13th International
Conference on Hydroinformatics

\title{
An Innovative ICT Solution for Sewer Systems
}

\author{
Antoni Grau ${ }^{1}$, Yolanda Bolea ${ }^{1}$, Alberto Sanfeliu ${ }^{2}$, Ana Puig-Pey ${ }^{2}$ \\ ${ }^{1}$ Technical University of Catalonia UPC, Pau Gargallo 5, 08028, Barcelona, Spain \\ ${ }^{2}$ Robotics Industrial Institute, UPC/CSIC, Llorens i Artigas 14, 08028 Barcelona, Spain \\ antoni.grau@upc. edu
}

\begin{abstract}
The objective of this paper is to explain the importance of research in wastewater transportation (sewage systems) using new technologies such as robotics systems and information and communication technologies (ICTs). ECHORD++ (European Coordination Hub for Open Robotics Development) is a very useful tool to foster this research and to meet needs and solutions. In this paper, authors explain the tool as well as the methodology to promote robotics research in urban environments, and the on-going experience will demonstrate that huge advances are made in this field.
\end{abstract}

\section{Introduction}

From their origins, cities have been the center of economic, political, urban, cultural and social innovation fed by growing flows of people seeking work and business opportunities and, finally, better life conditions. The growth of the cities in an ordered and planned way generated multiple challenges to the people that live, work or visit the city, but especially to those who manage and administrate it (Ontiveros, E., Vizcaíno, D. and López Sabater, V., 2016) (Smart Cities World, 2017). Europe has a long tradition of outstanding research and manufacturing in robotics. However, finding common ground between manufacturers and the research community has proven difficult in the past. Defining the future direction of robotics research has revealed to be the real challenge. European Coordination Hub for Open Robotics Development, ECHORD++ (ECHORD++, 2017), was installed as an incubator to promote innovation by facilitating the cooperation between academia and industry. ECHORD++ wants to further stimulate this interaction between robot manufacturers, researchers and users. Specifically, project managers will focus in PDTI (Public and users Driven Technological Innovation), that it is the methodology followed to develop a final technological product that is innovative and ready for commercialization (throughout the pairing academia-industry). Another key feature is that the product is not yet in the market and it is expected to be the solution for more than one cities. Regarding the PDTI side, ECHORD++ consists of different stages: initially, local authorities in city councils analyze the existing problems in the city that should be solved in a technological manner; each city can present a unique proposal in a public call; secondly, when all the presented proposals are collected a panel of experts score and rank them following some specific indicators (citizen's needs, social impact, 
sustainability impact, economic impact, efficiency...), only the best ranked proposal wins and this proposal will be "the challenge" to be solved; then, the proposal is refined by the winner city and presented, a public call is open to consortia (research centers and private companies) as a European project and only the two best ranked solutions are accepted to be working to solve the challenge. These two consortia are funded by ECHORD++ project to work in parallel for solving the challenge (Phase I, Phase II and Phase III) (Puig-Pey, A., Bolea, Y., Grau, A., and Casanovas, J., 2017). At the end of this process of 36 months, the suitable prototypes (evaluated by panel of experts- final reviewers) will be considered for industrial production and deployment.

\section{Material and Methods}

\subsection{The process of the project}

After a selection among 14 European cities that submit an innovative technological challenge, the city of Barcelona won the challenge in Urban Robotics with the project proposal "Robots for the inspection and the clearance of the sewer network in cities". After this selection an open call was performed by ECHORD++ administration and a total of 10 consortiums applied for the project. Finally, two consortiums were selected to develop their research in this challenge, ending up with a real prototype which will be presented at the end of the 36-month project. To develop this research project, proposers have to follow the Barcelona city regulations in this matter, Environmental Municipal Ordinance (1999). The new robotics solutions should include: 1) mobility and autonomy and 2) communication and teleoperation (ICTs). At the end of the project, the innovative technological solutions for the challenge of the Barcelona city proposed by both consortiums will be evaluated by a panel of experts, and it is expected that two robotics commercial products are the outcome of the project.

\subsection{The Challenge and The Need of Technological Solutions}

Sewer inspections require many people to work in risky and unhealthy conditions. Introducing a robotic solution in this process aims at reducing the labor risks, improving the precision of sewer inspections and optimizing sewer cleaning resources of the city. This system should be able to determine the state of the sewer in order to identify sewer segments where its functionality has been reduced either by sediments or by structural defects. Other functionalities required are sewer monitoring and water, air and sediment sampling. To well carry out these tasks, some general functions are required like remote operation, video and images capture, scanning and map building, communication and teleoperation, among others. Those items have to be approached with efficiency and in a sustainable manner. As we mentioned in the Introduction, two consortiums (SIAR, Sewer Inspection Autonomous Robot and ARSI, Aerial Robot for Sewer Inspection) have currently developing two possible technological solutions. The requirements for the new technology are given by the inherent sewer characteristics, that is, different ranges of pipe sizes, possible high concentration of, not explosive, but toxic gases as hydrogen sulphide, slippery areas, obstacles, atmosphere with $100 \%$ humidity, water temperature $16{ }^{\circ} \mathrm{C}$, and no telecommunication coverage in the sewer. The devices that could be considered as robots are able to move themselves in one direction by sewer and record video in $360^{\circ}$ to register the state of the sewer, they have to analyze the sewer by zooming navigating in $360^{\circ}$ by the video images, they need to access into the sewer system at one point and being recuperated in other point in an autonomous way, they are equipped with cameras, Laser, Lidar and Inertial navigation systems, Sonar sensor (for underwater detection if there is some stream of water) and hydrogen sulphide sensor, using a powerful communication system. With all data collected by the sensors, it is possible to generate a model of the interior of the sewer and identify the possible impairments. The improvements in the existing technology that this project seeks are to facilitate real-time decision making, innovation 
that makes inspection devices more autonomous, to have more degrees of freedom to move around the network, and the possibility to intensify the checking of a zone where impairment has been detected. The objective of developing this new technology is to mechanize sewer inspections in order to reduce the labor risks, objectify sewer inspections and optimize sewer cleaning expenses of the city. Regarding the sewer monitoring, the objective of sewer monitoring is to approximate the robot to the maximum level of sensitivity which will allow the sewer manager to make decisions without exposing to risky locations. The task of sample collecting is greatly important in order to obtain valid and traceable information, which could be used afterwards to determine environmental legislation and policies.

\section{Technological Results and Discussion}

The current need of the City of Barcelona is to mechanize sewer inspections in order to reduce the labor risks, objectify sewer inspections and optimize sewer cleaning expenses of the city. The sewer network of Barcelona is $1,532 \mathrm{~km}$ long, from pipe is at least $1.5 \mathrm{~m}$ high and workers are allowed to go inside it. In order to determine the state of the network, visual inspections are done with different frequencies depending on the slope and other characteristics of the sewer. Workers walk all along the pipe, in some sections even four times a year, and decide where it is necessary to clean. Moreover, sewers are classified as confined spaces which require special health and safety measures, in addition to other risks like slippery sections, obstacles or biological risks from the eventual contact with wastewater. These features made the process of sewer inspection a risky and expensive process that requires improvements urgently. Sewer inspection is a service included in the public management of the sewers of Barcelona. Nowadays, sewer inspections are done by people performing visual inspections and collecting information about the state of the sewage like sediment level and type, pipe obstructions, etc. Because of the sewer risks, the performance of the inspections is about $1.5 \mathrm{~km}$ of sewer every 6 hours. There are two technological solutions proposed (SIAR and ARSI projects) that are currently working and making progresses. Phase I of both projects has successfully showed the proposed solution of each one as proof of concept in the tests conducted in mobility, autonomy and communications. Currently these projects are in Phase II, during which all the aspects of the solution will be developed and validated to ensure a complete solution for robot-assisted sewer inspection. It is expected to obtain a prototype inspecting the Barcelona sewer network at the end this phase.

\subsection{SIAR Solution}

The SIAR solution seeks to solve the problem by the creation of an autonomous robot (see, Fig.1, left), with the possibility of control from a human operator in case of need of emergency. By using the proposed wireless system, augmented by the deployment of self-powered wireless repeaters, it will be possible to transmit bidirectional data between robot and an external operator, allowing the operator to change the mission in real time, instead of having to wait for the end of the sewer exploration to identify problems on the sewer system. The SIAR system will go beyond existing solutions through the inclusion of some innovative features, while maintaining affordable costs: configurable locomotion system with interchangeable wheels and tracks modules and variable length/width of the traction system; reliable navigation system based on data fusion from low-cost commercial RGB-D cameras, IMU and encoders, performing accurate localization and navigation on the sewer system. Communications rely on line of sight. As long as this line of sight is maintained the range and performance is satisfactory. The consortium intends to use repeaters to maintain communication beyond line of sight. It is recommended that the consortium evaluates the practicalities of deployment and retrieval of the repeaters in more detail as they may become a major impediment to practical application. Following the comments of the review, the consortium has been working (in Phase I) on the following aspects to analyze and improve the practicalities of the communication repeaters deployment/retrieval tasks: 1) Final design of the 
repeater package, including weight, batteries, autonomy and functionalities. 2) Design of the arm, including DoF (degrees of freedom), sizes, gripper and maximum payload. 3) Sensing approaches for estimating the relative position between robot and repeater for picking up the repeaters back to the robot (passive and active markers). The communication block will be equipped with a long range $2.4 \mathrm{GHz}$ router, the Microhard nVIP- 2400. This device has been tested indoors and proved to communicate up to 300 meters with line of sight. This link will provide a high bandwidth connection that could be used for video streaming and additional information such as the $3 \mathrm{D}$ reconstruction of the environment. With the proposed battery the repeater power autonomy will be close to 4 hours.
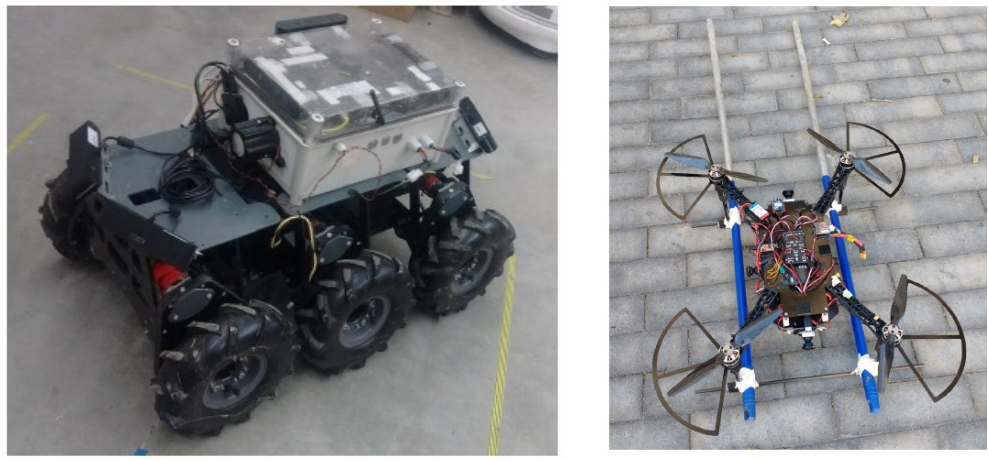

Fig. 1. (Left) SIAR and (Right) ARSI Prototypes

The deployment of repeaters is proposed whenever the Line-of-Sight (LoS) is expected to be lost, and therefore the quality of the links could begin to degrade. This will extend the operational range of the robot in more than $300 \mathrm{~m}$ for each repeater. To this end, the robotic platform will be equipped with a $4 \mathrm{DoF}$ manipulator on its back, which will automatically pick-up one of the available communication repeaters and deploy it on the ground (see Fig.2). This manipulator will also be used to retrieve the already deployed repeaters in its way back.
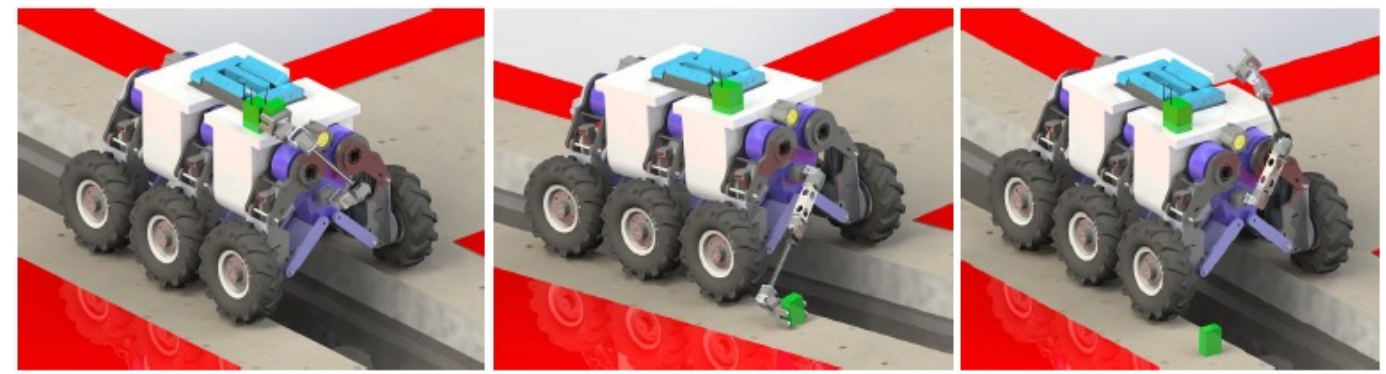

Fig. 2. SIAR Robot deploying one wifi communication repeater.

The robotic arm will be based on standard servo motors from Dynamixel (or similar). Currently, the arm components based on the required payload and workspace are under specifications. When completely extended the robotic arm will be able to reach a distance of $500 \mathrm{~mm}$. Its gripper will be based on an electromagnet which will be actuated during the deployment/collection of the repeaters (their enclosure will contain a metal part). A robust estimation of the pose of the repeaters is very important for their recovery using the manipulator. The design of the box will include passive AR tags for this. This would allow us to estimate the $6 \mathrm{DoF}$ relative position from the robot to the repeater. Some preliminary experimental results have been obtained by adding AR tags to the current repeaters made for Phase I. 


\subsection{ARSI Proposal}

The proposal is the use of a Micro Aerial Vehicle (MAV) (see Fig.1, right) for inspection tasks in the sewer avoids the mobility constraints from which a ground robot would suffer, such as paths with steps, steep drops and even objects in the way. Additionally, a flying platform is able to move faster through the sewer network than the terrestrial alternative and needs simpler logistics in deployment and operation. On the other hand, a MAV solution has to overcome strong constraints of size, weight and energy, as its flying space is bounded by sections less than $100 \mathrm{~cm}$ wide. Therefore, its size and consequently payload are limited to minimal dimensions. The ARSI consortium has addressed the problem of sewer inspection with the integral design of an aerial platform, multi-rotor type, endowed with sensors for semi-autonomous navigation and data collection within network, and capable of communicating with an on-surface operator. The different drones that will operating inside the sewer will be monitored and controlled from a van parked outside, in the street, and the workers will assist the robots to replace the batteries (Flying robots in sewers, 2015). Remote station in the sewer is used. The procedure aims at optimizing the range provided by our communications system and the autonomy of the batteries. To provide radio coverage in tunnel-like environments (tunnels, mines), two main methods are used: the leaky feeder, based on the use of radiating cables, and systems based on the natural propagation of radio-waves. Owing to the high cost of leaky-feeder installations and the fact that they are susceptible to damage and failure, the natural propagation system is preferred in many applications. Wireless propagation in these environments is described as strongly multipath, and if the wavelength of the signal is much smaller than the tunnel cross section, tunnels act as an oversized dielectric waveguide. In this case, the attenuation per unit length is low enough to allow communications over a range of up to several kilometers.

The main features of the design consist in: 1) Optimal layout of the payload on the platform (embedded PC, 2D laser scan, multiple image sensors, LED lights, gas sensor and antenna) which minimizes the weight and energy requirements. 2) Localization and motion planning methods in GPSdenied environments. 3) Careful design of the image sensors and their configuration in order to ensure a detailed view of the whole section for structure assessment and defect detection purposes. 4) Use morphology of the sewer network to enable out-of-sight communication. 5) Integration of mission planner within operational software of sewer maintenance companies.

The problem of the communications between the ARSI aerial vehicle and the Remote Station in the sewer was addressed and solved. Firstly, the challenge of the problem is described. As a result of a study of the problem and of the available technologies, the solution is described. The procedure aims at optimizing the range provided by our communications system and the autonomy of the batteries. To provide radio coverage in tunnel-like environments (tunnels, mines), two main methods are used: the leaky feeder, based on the use of radiating cables, and systems based on the natural propagation of radio-waves.

Owing to the high cost of leaky-feeder installations and the fact that they are susceptible to damage and failure, the natural propagation system is preferred in many applications. Wireless propagation in these environments is described as strongly multipath, and if the wavelength of the signal is much smaller than the tunnel cross section, tunnels act as an oversized dielectric waveguide. In this case, the attenuation per unit length is low enough to allow communications over a range of up to several kilometers. However, the signal is affected by strong fading phenomena, as has been studied by many authors (Dudley, D.G., Lienard, M., Mahmoud, S.F. and Degauque, P., 2007) (Molina-Garcia-Pardo, J.M., Lienard, M., Nasr, A., and Degauque, P., 2008). The environment that a sewer creates for telecommunications is challenging to say the least. Wired communications are not feasible given the aerial nature of our platform. Moreover, the multiple ground obstacles (corners, changes of level, water, etc.) present in sewers result in wired communications being impractical for almost any type of mobile platform. In the domain of wireless communications, only very low frequency signal travels without impediment in the underground. However, only high frequency waves provide the bandwidth and link 
performance necessary to meet the video and data streaming requirements of this project. The prototype has been flying in a real sewer environment, see Fig.3. The tests show that the behavior of the MAV is as expected.

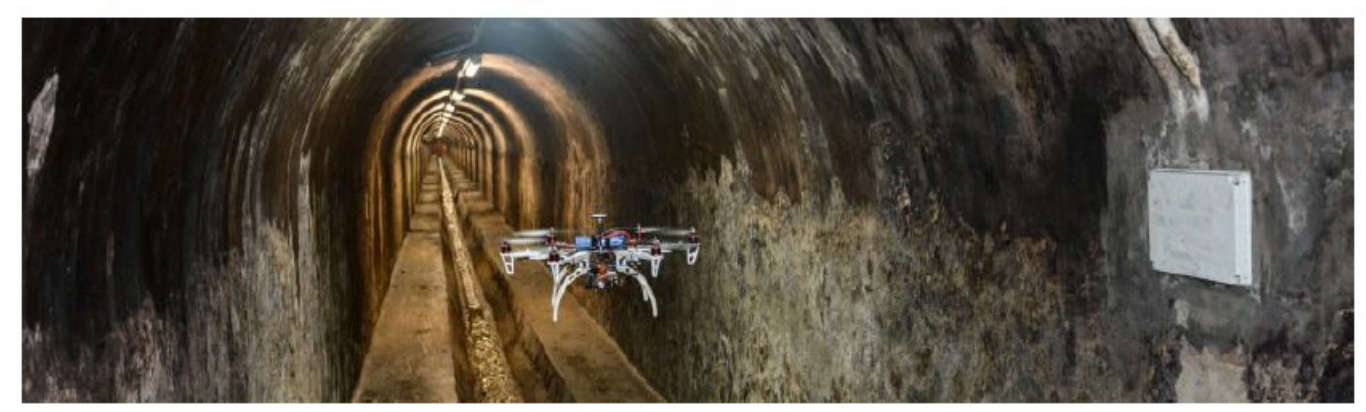

Fig. 3. Quadrator flight in Passeig Sant Joan in Barcelona, a real sewer environemnt.

\section{Conclusions}

In this paper, the challenge of sewage cleaning monitoring using robotics and communications is shown. Two selected consortia in the ECHORD++ carry out this challenge. The communication solution proposed in Phase I for both consortiums is explained obtaining satisfactory results. Next, in Phase II, a prototype (from the communication solution proposed in Phase I) will be presented. Then, both consortiums once again will be reviewed to pass Phase II.

\section{Acknowledgment}

Authors would like to thank the European Commission for launching ECHORD++ project. The "ECHORD++ EU Project FP7-ICT-2012-601116" project has received funding from the European Union's Seventh Framework Programme for research, technological development and demonstration.

\section{References}

Ontiveros, E., Vizcaíno, D. and López Sabater, V. (2016), Las ciudades del futuro: inteligentes, digitales y sostenibles; Telefonica Fundación, 2016, in Spanish.

Smart Cities World (2017), https://smartcitiesworld.net/news/news/rise-of-therobots-879, accessed on Oct 6th 2017.

ECHORD++ website (2017), http://http://echord.eu/, accessed on Oct 6th 2017.

Puig-Pey, A., Bolea, Y., Grau, A., and Casanovas, J. (2017) "Public entities driven robotic innovation in urban areas", Robotics and autonomous systems, Vol. 92, pp. 162-172, 2017.

Flying robots in sewers (2015) "El Pais" newspaper, December 7th, 2015,https://elpais.com/ccaa/2015/12/06/catalunya/1449424748_228383.html

Dudley, D.G., Lienard, M., Mahmoud, S.F. and Degauque, P. (2007) "Wireless propagation in tunnels", Antennas and Propagation Magazine, IEEE, vol. 49, no. 2, pages 11-26, April 2007.

Molina-Garcia-Pardo, J.M., Lienard, M., Nasr, A., and Degauque, P. (2008) "On the Possibility of Interpreting Field Variations and Polarization in Arched Tunnels using a Model for Propagation in Rectangular or Crcular Tunnels, IEEE Transaction on Antennas and Propagation, vol.56, issue 4, April 2008. 